\title{
Diet Congruent Primes - Are They Effective? The Effects of Food Priming on Restrained Eaters' Food Consumption
}

\author{
By Marie Bližkovská *
}

\begin{abstract}
This study examined the impact of healthy and unhealthy primes on the food choice of restrained and unrestrained eaters. Based on a literature review it was hypothesized that restrained eaters would be more susceptible to healthy primes. The reason for this hypothesis was that healthy primes would serve as a reminder of their diet and therefore promote healthy eating. To test the hypothesis, an experiment was carried out involving priming 92 subjects via posters with pictures of either healthy or unhealthy snacks. Participants were then asked about the food they purchased. The formulated hypothesis was confirmed since the results showed that indeed restrained eaters were more affected by the healthy prime than the unrestrained eaters. This result is in line with current studies. Moreover, unrestrained eaters were affected by the unhealthy prime, which was not the case for restrained eaters. Together these findings suggest that people are more responsive to primes that are congruent with their dietary constraints.
\end{abstract}

Keywords: Dieting reminders, Goal-conflict model, Healthy diet, Priming, Restrained eaters

\section{Introduction}

In recent years health awareness has become a very popular issue. People are starting to realize that a healthy lifestyle is an important and beneficial way of life. This phenomenon can be illustrated by the number of people on a diet. For instance, in 2008 the amount of restrained eaters in the USA alone rose to a 108 million people (Marketdata Enterprises Inc. 2012), accounting for $42 \%$ of the country's population. The growing awareness to food has had effects on public health alertness, as well as on markets and services associated with dieting. In economic terms, the reported annual increase in the diet related economic turnover in the USA in the year 2008 was $4.5 \%$ (Marketdata Enterprises Inc. 2012). This market includes anything from gyms to dieting programs to personal trainers. It is therefore safe to conclude that an increasing amount of people care about their health by reducing/keeping their body weight and take great measures to outline their aims as well as motivate themselves to achieve their goals. While people might genuinely have the intention to follow their diet and become healthier, their task is made almost impossible by subtle cues in their environment that invites them to eat unhealthy food and stray from their diet (Papies et al. 2014). A study that attempted to diminish the so-called yoyo effect found that a strikingly low amount of people are capable of maintaining their weight after initial weightloss over a longer period of time (Jeffery et al. 2000). It is evident that dieters are struggling to follow their set objectives, so this study will examine the effect of food primes on people's decision concerning food purchase and whether

${ }^{*}$ University of Valencia, Valencia, Spain. 
healthy primes can be used to promote healthy eating. The paper will also look at the relationship of priming and dieting on food consumption. This study is relevant because it can contribute to a better understanding of the relationship between market practices on the one hand and people's effort to eat healthier on the other.

As outlined before, healthy and dietetic goals are very hard to abide to. Maintaining or decreasing body weight is made even more difficult by the temptations and many obstacles highlighted by the literature. First of all, weight maintenance is associated with a strong willpower, determination, selfset goals and self-control, all traits that are very hard to develop (Elfhag and Rössner 2005). In addition to this, dieters must learn to resist temptations from advertisements or shops. In their paper Fedoroff et al. (2003) explain that they are harder to resist for people who are unsuccessful dieters. Houben et al. (2012) also found that unsuccessful dieters show more signs of disinhibition, as well as less inhibitory control over food-related responses. In a different study, participants who were presented with the smell of highly palatable and were then offered the same food consumed a larger amount of it than unrestrained eaters (Van Koningsbruggen et al. 2010). These factors in unsuccessful dieting account for the characteristics of the dieters, however there is more to it than only the personality traits of these people.

The overwhelming percentage of adults $(32.9 \%)$ and adolescents $(17 \%)$ in the USA that are obese can in part be explained by the environment we live in today (Ogden et al. 2007). As this study explains, an interaction between genetics and the obesogenic environment surrounding us has detrimental impacts on weight gain. Individuals who are genetically predisposed to crave highly palatable food respond to this environment in an even more dramatic fashion. A different study researching the impacts of easily accessible food on teenagers' weight found that readily accessible food contributes greatly to the consumption of unhealthy food (de Vet et al. 2013).

Looking at the literature, the concept of priming was identified as an important factor in the food temptation- food consumption pattern. As Fedoroff et al. (1997) explain, the growing amount of people who struggle to successfully remain on a diet might be clarified by the fact that a period of preexposure to a smell or image of food before eating can have an effect on an individuals' food consumption. The period of pre-exposure that they mentioned is referred to as a prime. Priming is an implicit concept activation which occurs after people have been exposed to a prime (Schacter 1992). It has been shown to work with stimuli that range anywhere from visual, olfactory, auditory to even semantic ones in and out of laboratory environments. Throughout the exposure, mental representations that are associated with the prime are activated in the participants' minds (Schnieder and Shriffrin 1997, Shriffrin and Schneider 1997). As mentioned before, external cues can greatly affect the consumption of food since the associated feelings of happiness, satisfaction, and fulfillment are made more available by the primes (Papies et al. 2015).

One research subject in literature studies how priming of dietetic goals can influence people's food consumption. Advertisements of food products on 
television or snacks presented at the cashier line in supermarkets and even olfactory cues are some of the everyday, ever present cues that restrained eaters must learn to ignore (Harris et al. 2009, Gaillet et al. 2013). Restrained eaters, people on a diet or obese people are especially susceptible to these primes (Papies et al. 2014, Fedoroff et al. 1997). One study used olfactory priming (the smell of fried chicken) in a shop, while free samples were offered. The researchers studied the amount of samples restrained and unrestrained participants ate after they had been primed with their dieting goals and they compared it to a condition where the dieting goals were not activated. In this experiment the prime had the form of a poster inviting participants to try a lowcalorie recipe. The results showed that restrained eaters are actually more vulnerable to food related priming than non-restrained eaters when they are not reminded of their dieting goals, causing them to consume more food (Papies and Hamstra 2010). Another study on dietary goals showed that restrained eaters that are overweight and are exposed to an extremely appetizing highcalorie prime forget about their dieting goals and instead focus on the food prime itself (Ouwehand and Papies 2010). Naturally these findings explain a dieters' inability to lose weight, but it also sheds a light on the gravity of the situation. Priming effects were found in experimental laboratory conditions, however the striking results are that priming is also occurring in non-controlled environments in our everyday life. That means that restrained eaters come into contact with, for them, extremely effective primes every day. Wadden et al. (2002) even describe obesity as an only natural response to a global epidemic of being constantly exposed to highly palatable foods, also called an obesogenic environment.

In another experiment, restrained participants that were primed with their dieting goals ate significantly less than their unprimed counterparts (Papies and Hamstra 2010). The mechanism behind this is thought to be the activation of unconscious processes that affect behavioral regulation. Such subtle reminders of a person's diet might then be enough to motivate them into regulating their eating behavior even in the food-rich environment (Papies and Veling 2013). The positive effect of subtle reminders of dieting was found even in overweight and obese people. On top of that it was shown that the prime does not have to be consciously processed to work (Papies et al. 2014).

In response to these findings, researchers have started to explore the idea of using primes to counter the effects of the environmental cues of the obesogenic environment we now live in. The obesogenic environment is defined as an environment that promotes gaining weight (Swinburn et al. 1999). Therefore, in the obesogenic environment of everyday life healthy dieting goal primes which are in conflict with it are difficult to encounter. Researchers thus looked for an alternative to the priming of dieting goal. The solution lies in using food primes which are a more frequent environmental cue.

Therefore, literature studied the effect that healthy food primes will have on the healthy diet. With this in mind Buckland et al. (2013a) chose to follow this line of reasoning. They did not prime the participants with their dietary goal directly, but rather through a healthy food prime. On one occasion 
participants were primed using a diet incongruent food - chocolate, and on the next occasion with a diet congruent healthy food - an orange, after which a snack was presented and the amount eaten was recorded. The prime did not have an effect on unrestrained eaters. However it did have a very positive effect on restrained eaters. When exposed to the orange, they ate $60 \%$ less chocolate compared to when they were primed with chocolate. This intervention might be more relevant for everyday struggles of restrained eaters, since food is a prime that one encounters more often than dieting primes. The method was thus fruitful as it helped restrained eaters eat less unhealthy food (chocolate) after being primed with healthy food (oranges) compared to restrained eaters that were primed with the unhealthy cue. As Buckland et al. (2014) point out, these results might have to do with the fact that diet congruent primes serve as reminders of dieting and thus reduce high-calorie food intake.

The experiment is a perfect example for the goal-conflict theory developed by Papies et al. (2008) and Stroebe et al. (2008), which states that dieters have two goals- succeeding in their diet, and the hedonic goal of eating palatable food. When exposed to for example chocolate, the hedonic goal will win, inhibiting the dieting goal from taking charge. This is what explains the results in Buckland et al. (2013a).

However, these results are almost contradictory to those of Ouwehand and Papies (2010)'s experiment. Here overweight and normal weight participants were primed with a tempting food. Subsequently, the craving of a high caloric food was assessed by presenting pictures of these foods. As they predicted, the exposure to diet incongruent food resulted in a decreased craving of it. A possible explanation of these results is that diet incongruent primes remind normal weight restrained participants of their diet, which leads them to consume more healthy food. Ouwehand and Papies (2010) state that this happens because the food is so opposite to their diet that they associate it with dieting and self-regulation. It is important to mention that Buckland et al. (2014) did not differentiate between normal weight and overweight participants. Moreover it is also possible that both results are true - that both extremely unhealthy and very healthy primes have the effect of reminding participants of their dieting goals.

There is evidence for both sides of the discussion. Buckland et al. (2013a) concluded that exposure to healthy food primes leads to healthy consumption. While Ouwehand and Papies (2010) argue that unhealthy food primes lead to healthy consumption. The second argument is strengthened by the findings of Wilcox et al. (2009) that showed that exposing participants to healthy and unhealthy food at the same time actually makes participants consume more unhealthy food. This is because participants, who are on a diet, feel like their goal to eat healthy is already fulfilled by just the mere presence of the healthy food, which allows them to indulge. At the same time, highly caloric food might just be too tempting to resist, as explained by the goal-conflict theory (Stroebe et al. 2008). 
As illustrated by the differing results above, the area of research dealing with diet congruent primes as reminders of dieting still does not fully understand the mechanisms behind it. It is therefore important to conduct more research in this area to understand the mechanisms behind people's choice and the role of food primes in it.

The aim of this article is to determine whether a diet congruent or diet incongruent prime can have an effect on people's subsequent choice of food. Previous research is ambivalent in this respect. However a majority of research suggests that healthy food primes will influence people to choose healthy food options (Papies and Veling 2013). Therefore our primary research hypothesis is that healthy food primes are successful in leading to choose a healthy food option.

An additional objective of this paper is to identify what role the interaction between exposure to food primes and the fact that a person is on a diet plays in choosing a healthy food. Therefore, the secondary hypothesis will test whether dieters are more susceptible to the healthy primes than non-restrained participants (Buckland et al. 2013a). The summary of the predicted hypotheses can be found in Figure 1. The model shows that it is predicted that healthy food primes will have a positive effect on healthy food choice, and the relationship will be moderated by diet. The unhealthy prime condition will have a negative effect on the food choice, and again the diet will be a moderator

While trying to figure out the effects of healthy and unhealthy food priming and to test the primary and secondary hypothesis a field experiment was carried out involving both dieters and non-dieters. This experiment is valuable for the field of psychology for three reasons. Firstly, it will provide clarity in the effect a prime has on the dietetic consumer pattern. Furthermore, methodologically it is a relevant and valuable study because it is a field study with a very generalizable sample of consumers which adds the aspect of external validity. The experiments in this area are mainly carried out in a laboratory setting. This research will therefore be able to determine whether the results found in those experiments hold in the field as well. As Wadden et al. (2002) explain, the percentage of obese people can be explained by the amount of highly palatable food always available around us. A better understanding of how these primes work outside of the laboratory setting could point towards possible solutions to the problem. Finally, the results of this study can thus have a consequent practical impact on how policymakers can most effectively encourage a healthy diet. This last reason has an obvious societal and economic implication. 
Figure 1. Model Based on Predicted Hypotheses

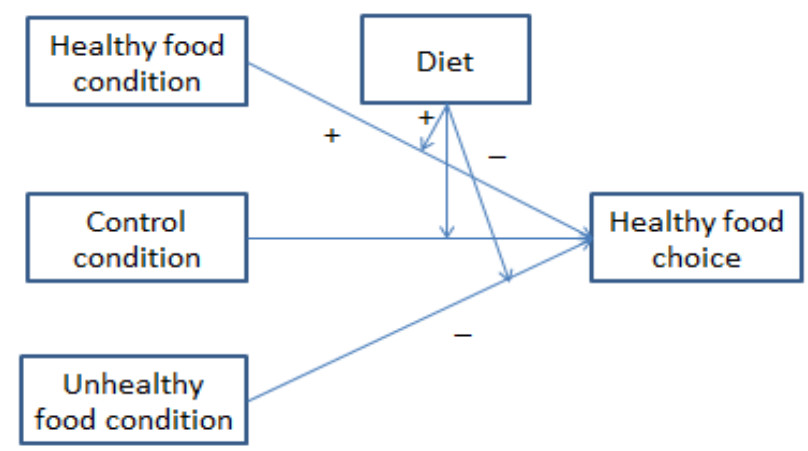

\section{Method}

\section{Participants}

The experiment was carried out at a sport stadium in the center of Utrecht (Netherlands) on weekend days in spring 2016. The participant pool therefore mainly included sport players (65.3\%), family members of the players $(18.9 \%)$, friends, fans $(10 \%)$ and others $(5.8 \%)$. Participants were obtained by a quota sampling method to provide us with an equal representation of both genders as well as the same number of participants per day. A total of 182 participants were questioned with an average of 60 participants per condition. The data however could not be used fully as it was not complete. Those participants who did not answer to either which food they purchased, or did not indicate their sex, age or whether they had a dietary constraint were excluded from the analysis. Using these criteria, $51.3 \%$ of the participants were left out of further analysis. Once the missing data was taken into account the average number of participants per condition became 31, resulting in 92 participants with complete data. All participants were aged over $18(M=32.7, S D=8.9)$ and signed an informed consent document. There were 43 females and 49 males in the final sample. The sample was consisted of 40 dieters and 52 non-dieters. Most participants had completed their Master studies (41.1\%), a large amount finished their Hbo (33.7\%), some were doing their Bachelor studies (14.7\%) and a small amount had an Mbo (10.5\%).

\section{Procedure}

The experiment was conducted on four separate days. One condition took two days to complete as there was a lack of available participants due to the events in the stadium. Every day was reserved for only one condition. This ensured that the participants were only exposed to one prime at a time. Each day the posters were hung up at all three entrances used by the customers before anyone came to the stadium. A basket of cookies and bananas with their price lay on the counter. Next the experimenters waited for the people to buy 
food before asking them to fill out a questionnaire. The questionnaire was filled out via internet, on phones, iPads and laptops, which was an incentive for people to join the study, as it is more enjoyable than pen-and-paper questionnaires and quicker. During the conversation with the participants the interviewers did not mention the presence of the primes or the exact purpose of the experiment, in order not to contaminate the responses.

\section{Materials}

During the experiment participants were asked to fill out a questionnaire in Dutch. As this was a field experiment it was not certain what group of people would be answering the questionnaire. It was thus imperative to make it understandable for all levels of education, nationalities and socio-economic groups. All the questions are therefore shorter and simpler versions of academically tested questionnaires on these subjects.

The questionnaire started off with questions about the participants' involvement in the stadium. Their reason for visiting and the frequency of their visits was asked by means of multiple choice questions. Next, the participants were asked about their usual food and drinks choices again via multiple choice questions. The multiple choice options were chosen based on the menu available in the canteen of the stadium. The following question was related to the dependent variable (food purchase). This was asked in the form of an open question. The reason for choosing an open question was to involve the participants more into the experiment. The free recall also ensured truthful responses, as people were not merely ticking options. They were also asked whether they had noticed anything different about the stadium canteen on the day of the experiment. We were wondering if participants noticed the posters with primes on the entrances. A mere $19 \%$ of the whole participant pool noticed a change in the environment that was connected to the experiment (usually the fruit and cookies on the counter).

After this, participants were asked about their diet in the form of three Likert scale questions. The questions were related to carefulness around calorie consumption, eating healthy and being vigilant about the food they eat. They were phrased in the following way: "People like me are careful about the amount of calories they eat every day". The items ranged from 1 to 7 , one meant completely disagree, and seven meant completely agree.

The last part of the questionnaire dealt with the demographic questions. Participants were inquired about their age, gender and education level (divided into Mbo, Hbo, WO (Bachelors), WO (Masters)). These questions were left to the end of the questionnaire as they could have affected people's responses, because of biases such as the self-fulfilling prophecy or stereotype threat.

\section{Measures}

The moderator variable that was examined was the dietary constraints of the participants. By dietary constraint we mean a self-constrained diet of 
people aiming at a healthier and well balanced way of eating. The variable had two categories: restrained eater and unrestrained eater. It was based on the answers participants gave on the three Likert scale questions on dietary constraints. The final measure was calculated as an average of the three responses. This was possible as the Cronbach's alpha was $\alpha=0.794$. The average of the three responses resulted in a continuous variable. The statistical tests that were employed, logistic regression and chi-squared, were however more suited to a dichotomous variable. The moderator variable was therefore divided along the median $(M D N=4.7)$, this helped create an equal pool of restrained and unrestrained eaters.

\section{Stimuli}

The experiment had a 3(control, healthy prime, unhealthy prime) $\mathrm{x}$ 2(restrained, unrestrained) factorial design with a hypothesized moderator of dieting (Figure 1). The experiment was a between subjects design.

The primes were administered by means of posters posted on all 3 entrances of the stadium. Based on a pilot study, a cookie was chosen to be the unhealthy prime and a banana as the healthy prime. This choice was made as the pilot study indicated that people clearly associated cookies with unhealthy and bananas with healthy foods. People described the bananas as "healthy" and "full of vitamins and minerals". The words used to describe the cookies were for example "filled with sugar" or "guilty pleasure". Since these were the associations that we wanted to prime the banana and cookie were appropriate choices. The only aspect of the poster that changed from condition to condition was the picture of the prime. The slogan "Snack of the week" was chosen as it was applicable to both of the primes. This resulted in the two posters to be found in Figure 2. The left picture shows the unhealthy food prime (cookie) and the right one shows the healthy prime (banana). The original size of the poster was an A4 paper format, placed at eye level. The third category was the control condition where no prime was shown. A control condition was necessary to determine the normal levels of the healthy and unhealthy food purchased in that location. The experimental conditions were then compared to the control condition to figure out the effect that former condition had. 
Figure 2. Pictures of the Posters that were used as Primes

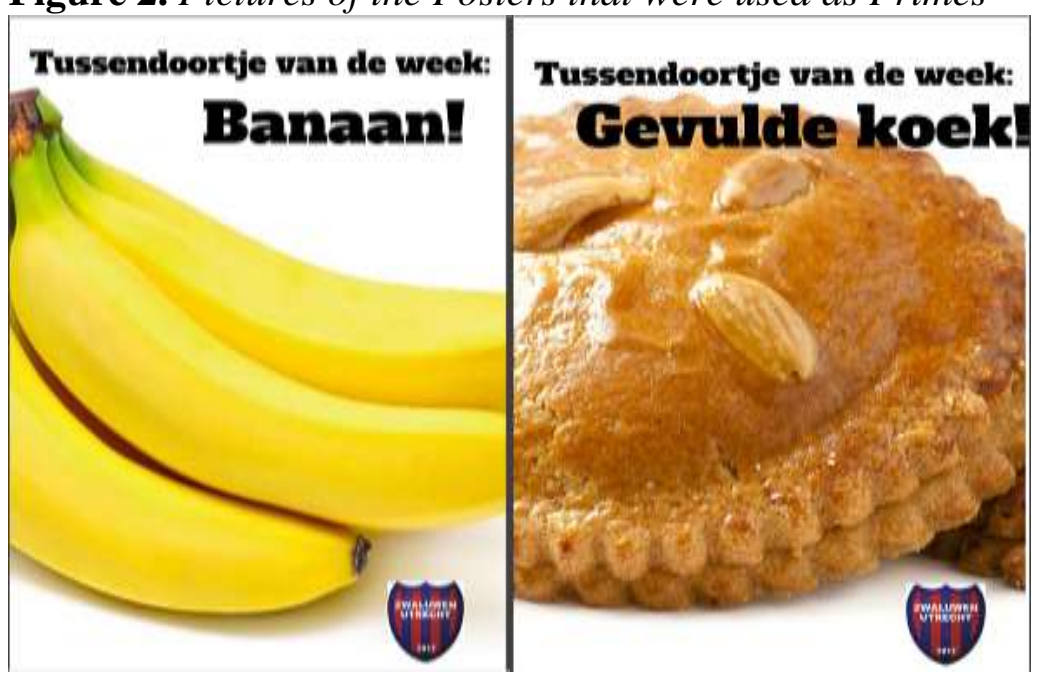

The dependent variable was defined as the food the participants chose to purchase in the cafeteria in the stadium. It had two nominal levels, buying healthy (banana) or buying unhealthy (cookie) food. The banana and cookie were primarily chosen as the target food which would serve as indicators of a healthy/unhealthy food choice. They have a similar price point allowing to charge the same amount of money for both of them $(0.50 €)$. They are both of a similar portion size and they are both snacks. They were an ideal choice as they were as similar as possible with the only thing different between them being their healthiness. However, only a very small percentage of participants bought these two snacks. So the entire menu of the canteen was coded by two independent experimenters into healthy and unhealthy options, and discussed the discrepancies to produce a dichotomous variable coded as healthy and unhealthy food purchased. If people bought both healthy and unhealthy food, the more prominent option was coded based on the number of items bought. An example of such an instance was a person buying fries (which were coded as unhealthy) as well as a coffee, "tosti" and a banana (all coded as healthy).

Analysis

A manipulation check was carried out to find out whether the manipulation worked correctly. It was however found that not a single one of the participants reported noticing the posters, which were used as primes in this context. Moreover this effect cannot be attributed to the fact that some people have only been at the stadium for the first time, since only $14.7 \%$ of the participants were there for the first time. More than half (53.7\%) visit the stadium every week and the rest come at least once a month, so they must know the surroundings very well, and would notice anything that is out of a place or new.

Moreover, a randomization check was run in order to find out whether all demographic variables were spread randomly across conditions. Since the ANOVA of condition per demographic variables was insignificant it could be concluded that the variables were in fact randomly distributed over the 
conditions. The variables of sex, gender and education level were therefore not incorporated into the following tests.

Two tests were used to analyze the data collected during the experiment. The first was a logistic regression test with condition and dieting as predictors. This method was used as it allows analyzing the relations between predictors and dichotomous dependent variables. The test offers statistical evidence for the significance and relevance of the variable, as well as the interaction effects between them. Because each variable was categorical, one of the categories served as the indicator against which the other categories were tested. For the conditions, the control condition was chosen as the indicator (coded as zero) which was then compared to the healthy condition (coded as one) and unhealthy condition (coded as two). In the dieting variable the non-dieters were the indicator. Unrestrained eaters were coded as one in the data set, and restrained eaters as two.

Secondly, a chi-squared test allowed for an investigation into the amount of participants in each condition and their food choice as well as whether they were on a diet or not. The chi-squared test was performed for the food purchased per condition, food purchased per dieting constraint and finally food purchased per condition as well as dieting constraint. The statistical program SPSS was used throughout the statistical analysis process.

\section{Results}

The primary research hypothesis related to the effectiveness of the two primes was tested using a logistic regression. The relevance and significance of each predictor in the model can be seen in Table 1 . The results of the logistic regression show that when only the prime was taken into account, $65.2 \%$ of the food choice was statistically correctly classified. The predictor that was most important in this model was the healthy condition $(\operatorname{Exp}(\beta)=0.260)$, as a significantly higher amount of participants chose the healthy food option (75\%) during this condition compared to the control condition (45.2\%). The unhealthy prime was not significant in predicting the distribution of the food choice $(\operatorname{Exp}(\beta)=1.647)$. The difference between the healthy $(33.3 \%)$ and unhealthy $(66.7 \%)$ food purchase during the unhealthy food prime was very similar to the difference in the purchase of healthy $(45.2 \%)$ and unhealthy $(54.8 \%)$ food in the control condition (for more results see Table 2). In other words, the unhealthy prime was therefore not an effective prime in stimulating people to buy a particular food. The unhealthy prime was comparable to the control condition, meaning that it did not influence the consumers' choice. On the other hand the healthy prime proved to differ from the control condition and thus proved to influence people's food choice. 
Table 1. Coefficients in Logistic Regression of Food Purchase Predicted by Conditions and Restrained Eating

\begin{tabular}{|c|c|c|c|c|c|c|c|c|}
\hline & \multirow[t]{2}{*}{$\bar{\beta}$} & \multirow[t]{2}{*}{ S.E. } & \multirow[t]{2}{*}{ Wald } & \multirow[t]{2}{*}{ df } & \multirow[t]{2}{*}{ Sig. } & \multirow[t]{2}{*}{$\operatorname{Exp}(\beta)$} & \multicolumn{2}{|c|}{$\begin{array}{c}\text { 95\% C.I. for } \\
\operatorname{Exp}(\beta)\end{array}$} \\
\hline & & & & & & & Lower & Upper \\
\hline \multicolumn{9}{|l|}{ Step 1} \\
\hline Control & - & - & 10.035 & 2 & 0.007 & - & - & - \\
\hline Healthy condition & -1.347 & 0.591 & 5.189 & 1 & 0.023 & 0.260 & 0.082 & 0.829 \\
\hline Unhealthy condition & 0.499 & 0.505 & 0.975 & 1 & 0.323 & 1.647 & 0.612 & 4.434 \\
\hline Constant & 0.194 & 0.361 & 0.289 & 1 & 0.591 & 1.214 & - & - \\
\hline \multicolumn{9}{|l|}{ Step 2} \\
\hline Control & - & - & 5.068 & 2 & 0.079 & - & - & - \\
\hline Healthy condition & 0.000 & 0.935 & 0.000 & 1 & 1.000 & 1.000 & 0.160 & 6.255 \\
\hline Unhealthy condition & 1.609 & 0.780 & 4.258 & 1 & 0.039 & 5.000 & 1.084 & 23.061 \\
\hline $\begin{array}{l}\text { Healthy condition by } \\
\text { restrained }\end{array}$ & -2.216 & 1.238 & 3.203 & 1 & 0.074 & 0.109 & 0.010 & 1.235 \\
\hline $\begin{array}{l}\text { Unhealthy condition } \\
\text { by restrained }\end{array}$ & -2.098 & 1.050 & 3.993 & 1 & 0.046 & 0.123 & 0.016 & 0.961 \\
\hline Restrained eaters & 0.894 & 0.741 & 1.455 & 1 & 0.228 & 2.444 & 0.572 & 10.448 \\
\hline Constant & -0.288 & 0.540 & 0.284 & 1 & 0.594 & 0.750 & - & - \\
\hline
\end{tabular}

Note: Step1: Model: $\chi^{2}(2, n=92)=11.428, p=0.003$.

Step 2: Model: $\chi^{2}(5, n=92)=17.454, p=0.004$.

From this part of the analysis it could be concluded that participants who saw the healthy prime chose healthier food. Those who saw the unhealthy prime or were in the control condition did not change their food choice. Their actions were not affected by the prime.

Moving to the secondary research hypothesis which tested the interaction effect between the dieting constraints and the conditions, to which the participants were exposed to, we first looked at the moderator variable alone. The moderator diet variable by itself in the logistic regression was not significantly important in the prediction of participants' food choice $(\operatorname{Exp}(\beta)=2.444)$. As can be understood from Table 3, overall participants on a diet bought almost the same amount of healthy food $(55.8 \%)$ as unhealthy food $(44.2 \%)$. Additionally, the ones who indicated that they were not on a diet bought more unhealthy food $(60 \%)$ than healthy food $(40 \%)$. It can then conclude that people on a diet did not buy significantly different food from people who were not on a diet. Diet by itself does not affect food purchase.

Table 2. Chi-Squared Distribution of Food Purchased per Condition

\begin{tabular}{|c|c|c|c|c|c|c|}
\hline & \multicolumn{3}{|c|}{ Condition } & \multirow[b]{2}{*}{ Tota } \\
\hline & & & Control & Healthy & Unhealthy & \\
\hline \multirow{4}{*}{$\begin{array}{l}\text { Purchased } \\
\text { Food }\end{array}$} & \multirow{2}{*}{$\begin{array}{l}\text { Healthy food } \\
\text { choice }\end{array}$} & Count & 14 & 21 & 12 & 47 \\
\hline & & $\begin{array}{l}\% \text { within } \\
\text { condition }\end{array}$ & 45.2 & 75.0 & 33.3 & 49.5 \\
\hline & \multirow{2}{*}{$\begin{array}{l}\text { Unhealthy } \\
\text { food choice }\end{array}$} & Count & 17 & 7 & 24 & 48 \\
\hline & & $\begin{array}{l}\% \text { within } \\
\text { condition }\end{array}$ & 54.8 & 25.0 & 66.7 & 50.5 \\
\hline \multirow{2}{*}{\multicolumn{2}{|c|}{ Total }} & Count & 31 & 28 & 36 & 95 \\
\hline & & $\begin{array}{l}\% \text { within } \\
\text { condition }\end{array}$ & 100.0 & 100.0 & 100.0 & 100.0 \\
\hline
\end{tabular}


The next set of results pertaining to the secondary hypothesis dealt the interaction between the primes and diet on the food choice. This was tested using the Logistic regression which classified $67.4 \%$ of the participants correctly when the diet variable was added. The model was thus improved and explained more of the variance when dieting was included.

Table 3. Chi-Squared Distribution of Food Purchased per Dietary Constraint

\begin{tabular}{|c|c|c|c|c|c|}
\hline & \multicolumn{2}{|c|}{ Diet } & \multirow[t]{2}{*}{ Total } \\
\hline & & & $\begin{array}{c}\text { Unrestrained } \\
\text { eaters }\end{array}$ & $\begin{array}{c}\text { Restrained } \\
\text { eaters }\end{array}$ & \\
\hline \multirow{4}{*}{$\begin{array}{l}\text { Purchased } \\
\text { Food }\end{array}$} & \multirow{2}{*}{$\begin{array}{l}\text { Healthy food } \\
\text { choice }\end{array}$} & Count & 16 & 29 & 45 \\
\hline & & $\begin{array}{l}\text { \% within } \\
\text { diet }\end{array}$ & 40.0 & 55.8 & 48.9 \\
\hline & \multirow{2}{*}{$\begin{array}{l}\text { Unhealthy food } \\
\text { choice }\end{array}$} & Count & 24 & 23 & 47 \\
\hline & & $\begin{array}{l}\% \text { within } \\
\text { diet }\end{array}$ & 60.0 & 44.2 & 51.1 \\
\hline \multirow{2}{*}{\multicolumn{2}{|c|}{ Total }} & Count & 40 & 52 & 92 \\
\hline & & $\begin{array}{l}\% \text { within } \\
\text { diet }\end{array}$ & 100.0 & 100.0 & 100.0 \\
\hline
\end{tabular}

A chi-squared test showed that participants on a diet who were subjected to the unhealthy condition bought significantly different food than those who were not on a diet $(\operatorname{Exp}(\beta)=0.123)$. Restrained eaters bought a similar amount of healthy food $(47.1 \%)$ as unhealthy food $(52.9 \%)$, while unrestrained eaters bought less healthy food $(21.1 \%)$ than unhealthy food $(78.9 \%)$. An overview of these results can be found in Figures 3 and 4. It can be observed that in the unhealthy condition unrestrained eaters purchase more unhealthy food than healthy food. In the healthy condition the count is comparable, and in the control condition the level of healthy food purchased is higher than unhealthy food purchased (Figure 3). In the control condition more people bought unhealthy food. In the healthy condition the healthy food was more prominent than in the unhealthy food. In the unhealthy condition the levels are comparable (Figure 4). In other words, restrained eaters were not affected by the unhealthy prime as much as the unrestrained eaters were. Moreover, the unhealthy prime swayed participants choice towards buying unhealthy food.

In the healthy condition the purchase of restrained and unrestrained eaters were less different resulting in a marginally significant result $(\operatorname{Exp}(\beta)=0.109)$. Here the unrestrained participants bought an almost equal amount of healthy $(57.1 \%)$ and unhealthy food $(42.9 \%)$ as opposed to the restrained participants who purchased a higher number of healthy food (83.3\%) than of unhealthy food $(16.7 \%)$. In this situation the restrained eaters were more susceptible to the prime than the unrestrained eaters, but both groups bought overall more healthy food. 
Figure 3. The Frequency Distribution of Purchase for Unrestrained Eaters per Condition

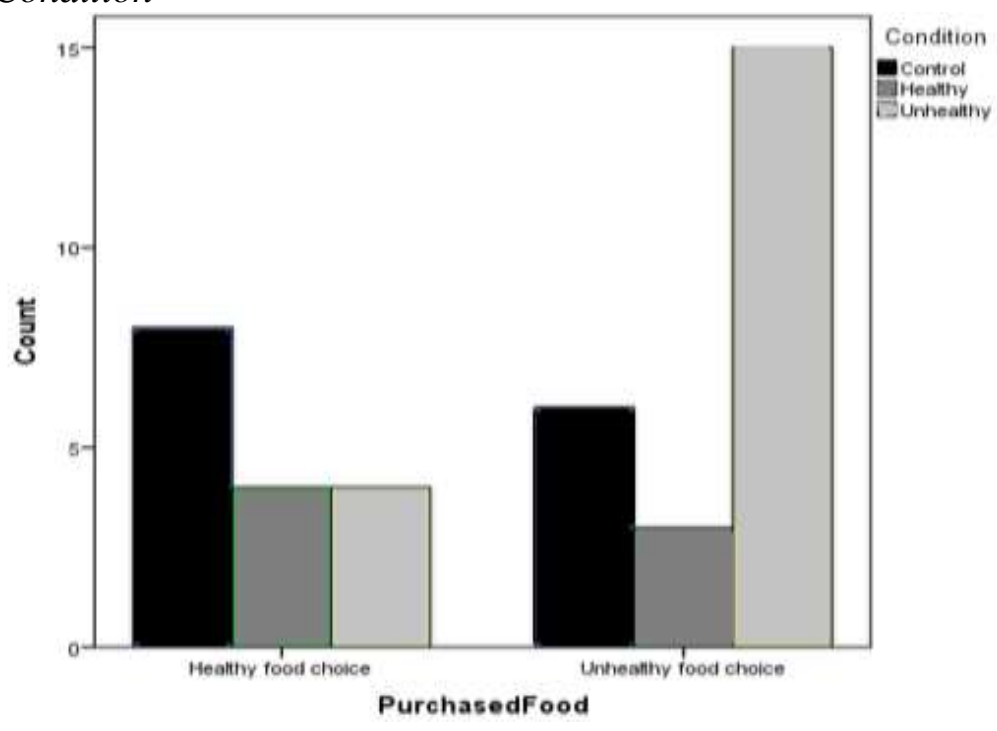

Figure 4. The Frequency Distribution of Purchase for Restrained Eaters per Condition

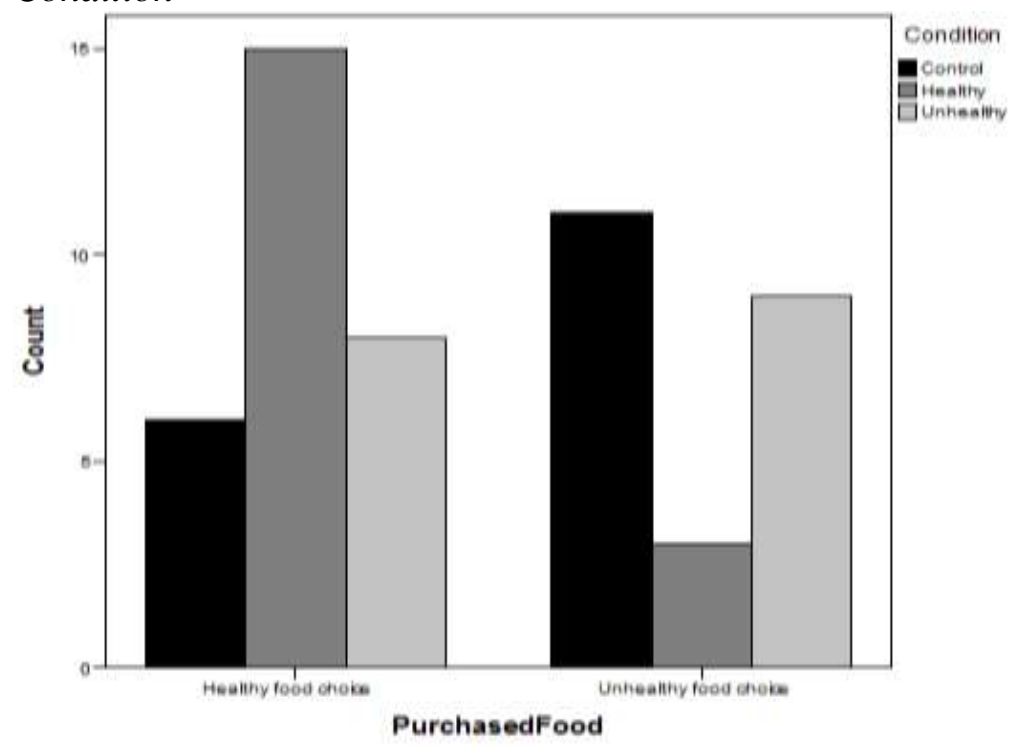

It is also worth noting that in the control condition the unrestrained eaters purchased an almost equal amount of healthy (57.1\%) and unhealthy (42.9\%) food, whereas the restrained eaters purchased much more unhealthy food $(64.7 \%)$ when no prime was shown than healthy food $(35.3 \%)$. This indicates that if no prime is used the environment of the experiment (stadium) naturally influences restrained eaters to be more inclined to buy unhealthy food. The opposite is true for the unrestrained eaters who tend to buy more healthy food in the event that no prime was shown. 


\section{Discussion}

The present study was conducted in order to determine whether a healthy food prime can encourage a person's choice of food to be healthy as well as what role dieting has in this relationship. The primary hypothesis that the experiment was trying to test was whether healthy food primes are effective in promoting healthy food choice. The results confirm the primary research hypothesis, namely that healthy food primes are able to influence people's choice towards healthy food. Participants were more prone to buying healthy products when exposed to a healthy prime compared to the control or the unhealthy prime conditions. It was also hypothesized that participants on a diet will be more susceptible to the healthy primes since the healthy prime serves as a reminder of their diet, which leads them to choose products that are within their diet. The analysis provided evidence for the effectiveness of the healthy prime on dieting participants, so the secondary hypothesis could also be accepted. The results suggest a relationship between the healthy prime and restrained eaters, since they purchased more healthy food during the healthy prime than the unrestrained eaters. Other results, not concerning either hypothesis surged from the analysis. Restrained eaters were not affected by the unhealthy prime at all. Unrestrained eaters were more susceptible to the unhealthy prime and bought more unhealthy products when exposed to the unhealthy prime compared to the healthy prime. Interestingly, the data from the control condition tell us that the restrained eaters buy more unhealthy products than the unrestrained eaters.

The primary hypothesis concerning the effect of the prime on food choice was confirmed by the following results. Participants were affected by the healthy prime since they chose more healthy food when exposed to the prime, in comparison to the control or the unhealthy prime condition. This result confirms previously known notions about environmental primes or cues that people encounter in their everyday life (Forwood et al. 2015, Stöckli et al. 2016). As Bargh (2006) explains, there are pre-existing associations between concepts and objects that determine which cue will be involved in the decision in a food related situation, and this result confirms this link. Moreover, as was expected, the healthy prime enhanced healthy purchases. The results follow the trend of previous findings in this area, showing that food or diet related primes have an effect on people's eating constraints (Buckland et al. 2013b, Papies and Veling 2013). All of these papers show that a pre-exposure to healthy food has a positive effect on the following food consumption.

Concerning the secondary hypothesis the following can be considered. The moderator variable of dieting yielded interesting results that are partly in line with the formulated hypothesis and provide even more insight into the issue of dieting and susceptibility to priming. As was predicted, the healthy prime was more efficient at promoting healthy food choices for dieters. The same prime was however ineffective for unrestrained eaters. These results replicate those of Buckland et al. (2013a) perfectly. They primed restrained and unrestrained participants with healthy food, leading them to consume less food. The prime 
was only effective for restrained participants, results which have also been found by the present study. Although, it must be taken into account that Buckland et al. (2013a) measured the amount of intake, while this study measured food choice. More research must take place before a generalized conclusion can be attained, because the present results were only marginally significant, and both studies tested a different dependent variable.

The same moderator produced very different results when it came to the unhealthy food prime. Here it was only effective in leading unrestrained people to choose the less healthy of the two options. Restrained eaters were not affected by this prime as it did not push them towards unhealthy food nor to the healthy food. The fact that the unhealthy prime did not push restrained eaters towards eating healthier therefore challenges the goal-conflict model put forward by Papies et al. (2008) and Stroebe et al. (2008), mentioned in the introduction. The model proposes that when a palatable food is presented to a restrained eater it activates their hedonic goal to eat the highly enjoyable food (Lowe and Butryn 2007), which then inhibits the dieting goal. The restrained eaters in the unhealthy condition however fail to show that effect. In this experiment, the unhealthy prime did not have any effect on the restrained eaters, suggesting that restrained eaters in a way block their minds from noticing highly palatable food, which could ruin their diet. This reasoning would also explain why the results are not in line with those of Ouwehand and Papies (2010), where the exposure to unhealthy food leads restrained eaters to eat healthier as the prime triggers dieting goals because what the participants see is so opposite to their diet that it reminds them of it. On the other hand the second result concerning the unrestrained eaters in the unhealthy condition, who were affected by the prime, is very much in line with the goal-conflict model. This is because in this case there is no goal conflict meaning that for the unrestrained eaters the highly palatable food only activates their hedonic goal which they also follow. These differing results show that the goal-conflict model which is commonly used to explain the mechanisms of priming restrained eaters does not apply to the situation at hand.

One way to explain the results in a way that incorporates all of them is by saying that people are more aware of primes that are in line with their dietary constraints. Restrained eaters notice healthy primes more because they are on the lookout for anything that is linked to their diet (Buckland 2013). Unrestrained eaters on the other hand are more alert for primes and cues concerning the unhealthy lifestyle such as snacks. There is a lack of research looking into the effects that food primes have on unrestrained eaters. This explanation is however viable since people are more aware of the primes that are congruent to their diet, they are also more affected since they notice them more often. Because the primes are in line with their diet they are more personal and can have a bigger more personal effect on the observers. It could therefore be summarized that people are more affected by primes that are congruent with their own diet.

Another notable conclusion that can be reached based on these results is that the primes used in this experiment worked at an unconscious level, at the 
so-called subliminal level. A very small amount of people noticed changes and none of them noted any changes pertaining to the posters. A similar result was shown by a number of previous studies, where primes of all natures were responsible for a change in behaviors or attitudes, without having been processed consciously (Custers and Aarts 2007). There is evidence of unconscious processing of the primes since there was a significant effect of the primes which means that the primes must have been noticed, remembered and processed by the participants.

The experiment designed for this study has a number of strengths. Probably the most important one is the naturalistic setting of the experiment. It was carried out in an environment that is closer to real life than the laboratory setting that is usually employed. The sample was also representative of the general population, since both genders were equally distributed over the conditions, and the age and education level was spread over a wider range. This is typically not the case as research is mainly carried out in college settings where the age and education level is uniform. Such a homogeneous and unrepresentative sample means that the results cannot be generalized to the rest of the population. The difference in result that two samples can yield is apparent from the following example in the field of priming. According to Forwood et al. (2015), the level of a person's education has an effect on how susceptible they are to primes. Those who are more educated were more influenced by healthy priming than less educated people. The present study also builds upon experiments that do not research both genders (Anschutz et al. 2008) and adds generalizability to the studies that chose to benefit from the controlled environments of laboratory settings (Fishbach et al. 2003).

Concerning the limitations of this study, it is clear that unfortunately, the naturalist setting comes with a price. The fact that there was less control over the participants meant that more participants did not fill out the entire questionnaire and people who had not eaten yet were asked to participate. Moreover, the participants were asked to recall what they had eaten in the day, which can be hard to do and a lot of little snacks can be forgotten. All of this contributed to the large amount of missing data. Additionally, in the case of this study it is also hard to figure out which of the missing data is due to the fact that participants had not eaten yet, and which of them ignored the question. While the sample size diminished quite a lot, the average per condition remains above 30. Therefore, a normal distribution can be assumed making it possible to employ normal statistical methods for the analysis (Corder and Foreman 2009). The problem of the lack of power due to the missing data of the banana and cookie purchase was resolved by including all the food that was purchased into the analysis. In this way the validity of the experiment remained high and the amount of data increased as well. More importantly however the naturalist settings meant that the participants were not acquainted with experiments or questionnaires. A number of them were not familiar with the Likert scale questions; others were discussing answers amongst each other. An experimenter was always close, to answer any questions or stop unfavorable behavior, but the conditions were not optimal. 
Another limitation which was not taken into account was whether participants were thirsty or hungry while taking part in the experiment. In a recent study hunger and thirst were found to have an important interaction effect with dieting and priming, this should therefore be incorporated into the study (Forwood et al. 2015). For practical reasons, the questionnaire was kept as short and comprehensive as possible so everybody could understand it and be able to answer all questions without problems. However, more research could focus on this aspect to reproduce the results as this is a very new field of research.

Finally, it is important to point out that the location of the experiment was a sport stadium which in itself might have been a prime for healthy life choices. Furthermore, the canteen did not offer as many healthy food choices as unhealthy food choices, so when the bananas were introduced it could have had an unforeseen effect on the participants. The effect of both of these factors was however examined during the control condition. The unrestrained eaters purchased an equal amount of healthy and unhealthy food, so the environment did not affect them. The restrained eaters ate more unhealthy food. It is possible that the sporty environment made them feel as if they had already done something for their diet that day and they could now "cheat". This phenomenon has been reported in literature as compensatory health beliefs (Radtke et al. 2012). It refers to the behavior model where people are more inclined to partake in unhealthy behaviors because they are persuaded that an upcoming healthy behavior can overrule the effects of the unhealthy one. Further research could test whether experiments in other locations would come to the same conclusions.

\section{Conclusion}

This study set out to increase knowledge on the topic of priming of restrained eaters within the field of health psychology. It attempted to understand the effects of a prime and whether food could serve as a reminder of people's diets as well as the role people's diets have in prime effectiveness. This question was answered by means of an experiment in which participants were primed by healthy and unhealthy food posters and their subsequent food purchase was studied. Especially the healthy prime did have an effect on the food choice, confirming the primary hypothesis. But the more interesting result was that in fact restrained eaters were more affected by healthy food primes, buying healthier products, and unrestrained eaters were more affected by the unhealthy prime causing them to buy unhealthy products. While both results follow the secondary hypothesis, they are simultaneously evidence for and against the goal-conflict model which this study was trying to disprove. A proposed explanation for the present results was that people are more aware and therefore more affected by primes that are congruent with their own personal diet. A deeper exploration of the current research question using more resources and better defined food choices would then be greatly beneficial to 
the current development of this field of research. Moreover, new research is still necessary in this field that would focus on the concepts of thirst and hunger and as well as research that would elaborate on the issue of the effects that primes have on the choice of food in different locations and environments. A better understanding of this area is needed and every new theory or model helps with the problem at hand - staying healthy in the tempting world we live in today.

\section{References}

Anschutz DJ, Van Strien T, Engels RCME (2008) Exposure to slim images in mass media: Television commercials as reminders of restraint in restrained eaters. Health Psychology 27: 401-408.

Bargh JA (2006) What have we been priming all these years? On the development, mechanisms, and ecology of nonconscious social behavior. European Journal of Social Psychology 36(2): 147-168.

Buckland NJ (2013) The role of diet-congruent cues in short term food intake (Doctoral dissertation, University of Leeds).

Buckland NJ, Finlayson G, Hetherington MM (2013a) Pre-exposure to diet-congruent food reduces energy intake in restrained dieting women. Eating Behaviors 14(3): 249-254.

Buckland NJ, Finlayson G, Hetherington MM (2013b) Slimming starters. Intake of a diet-congruent food reduces meal intake in active dieters. Appetite 71: 430-437.

Buckland NJ, Finlayson G, Edge R, Hetherington MM (2014) Resistance reminders: Dieters reduce energy intake after exposure to diet-congruent food images compared to control non-food images. Appetite 73: 189-196.

Corder GW, Foreman DI (2009) Comparing two related samples: The Wilcoxon signed ranks test. Nonparametric Statistics for Non-Statisticians: A Step-by-Step Approach: 38-56.

Custers R, Aarts H (2010) The unconscious will: How the pursuit of goals operates outside of conscious awareness. Science 329(5987): 47-50.

de Vet E, de Wit JB, Luszczynska A, Stok FM, Gaspar T, Pratt M, de Ridder DT (2013) Access to excess: how do adolescents deal with unhealthy foods in their environment?. The European Journal of Public Health 18: 93-112.

Elfhag K, Rössner S (2005) Who succeeds in maintaining weight loss? A conceptual review of factors associated with weight loss maintenance and weight regain. Obesity Reviews 6(1): 67-85.

Fedoroff ID, Polivy J, Herman CP (1997) The effect of pre-exposure to food cues on the eating behavior of restrained and unrestrained eaters. Appetite 28(1): 33-47.

Fedoroff I, Polivy J, Herman CP (2003) The specificity of restrained versus unrestrained eaters' responses to food cues: general desire to eat, or craving for the cued food?. Appetite 41(1): 7-13.

Fishbach A, Friedman RS, Kruglanski AW (2003) Leading us not unto temptation: Momentary allurements elicit overriding goal activation. Journal of Personality and Social Psychology 84: 296-309.

Forwood SE, Ahern AL, Hollands GJ, Ng YL, Marteau TM (2015) Priming healthy eating. You can't prime all the people all of the time. Appetite 89: 93-102. 
Gaillet M, Sulmont-Rossé C, Issanchou S, Chabanet C, Chambaron S (2013) Priming effects of an olfactory food cue on subsequent food-related behavior. Food Quality and Preference 30(2): 274-281.

Harris JL, Bargh JA, Brownell KD (2009) Priming effects of television food advertising on eating behavior. Health Psychology 28(4): 404-410.

Houben K, Nederkoorn C, Jansen A (2012) Too tempting to resist? Past success at weight control rather than dietary restraint determines exposure-induced disinhibited eating. Appetite 59(2): 550-555.

Jeffery RW, Epstein LH, Wilson GT, Drewnowski A, Stunkard AJ, Wing RR (2000) Long-term maintenance of weight loss. Current status. Health Psychology 19: 5-16.

Lowe MR, Butryn ML (2007) Hedonic hunger: A new dimension of appetite?. Physiology \& Behavior 91: 432-439.

Marketdata Enterprises Inc. (2012). Number of American Dieters Soars to 108 Million; Market to Grow 4.5\% to \$65 Billion in 2012 (Press Release). Tampa: 2-4.

Ogden CL, Yanovski SZ, Carroll MD, Flegal KM (2007) The epidemiology of obesity. Gastroenterology 132(6): 2087-2102.

Ouwehand C, Papies EK (2010) Eat it or beat it. The differential effects of food temptations on overweight and normal-weight restrained eaters. Appetite 55(1): 56-60.

Papies EK, Hamstra P (2010) Goal priming and eating behavior: enhancing selfregulation by environmental cues. Health Psychology 29(4): 384-388.

Papies EK, Veling H (2013) Healthy dining. Subtle diet reminders at the point of purchase increase low-calorie food choices among both chronic and current dieters. Appetite 61: 1-7.

Papies EK, Barsalou LW, Press G (2015) Grounding desire and motivated behavior: A theoretical framework and review of empirical evidence. The Psychology of Desire 4: 36-60.

Papies EK, Potjes I, Keesman M, Schwinghammer S, Van Koningsbruggen GM (2014) Using health primes to reduce unhealthy snack purchases among overweight consumers in a grocery store. International Journal of Obesity 38(4): 597-602.

Papies EK, Stroebe W, Aarts H (2008) Understanding dieting: A social cognitive analysis of hedonic processes in self-regulation. European Review of Social Psychology 19: 339-383.

Radtke T, Scholz U, Keller R, Hornung R (2012) Smoking is ok as long as I eat healthily: Compensatory health beliefs and their role for intentions and smoking within the health action process approach. Psychology \& Health 27(2): 91-107.

Schacter DL (1992) Implicit knowledge - New perspectives on unconscious processes. Proceedings of the National Academy of Sciences of the United States of America 89: 11113-11117.

Schneider W, Shiffrin RM (1977) Controlled and automatic human informationprocessing -1- detection, search, and attention. Psychological Review 84: 1-66.

Shiffrin RM, Schneider W (1977) Controlled and automatic human informationprocessing -2- perceptual learning, automatic attending, and a general theory. Psychological Review 84: 127-190.

Stöckli S, Stämpfli AE, Messner C, Brunner TA (2016) An (un)healthy poster: When environmental cues affect consumers' food choices at vending machines. Appetite 96: 368-374.

Stroebe W, Mensink W, Aarts H, Schut H, Kruglanski AW (2008) Why dieters fail: Testing the goal conflict model of eating. Journal of Experimental Social Psychology 44: 26-36. 
Swinburn B, Egger G, Raza F (1999) Dissecting obesogenic environments: the development and application of a framework for identifying and prioritizing environmental interventions for obesity. Preventive Medicine 29(6): 563-570.

Van Koningsbruggen GM, Stroebe W, Aarts H (2010) Successful dieting in tempting environments: Mission impossible. Mind Magazine 11: 132-139.

Wadden TA, Brownell KD, Foster GD (2002) Obesity. Responding to the global epidemic. Journal of Consulting and Clinical Psychology 70: 510-525.

Wilcox K, Vallen B, Block L, Fitzsimons GJ (2009) Vicarious goal fulfillment: When the mere presence of a healthy option leads to an ironically indulgent decision. Journal of Consumer Research 36(3): 380-393. 